\title{
EFFECT OF DILTIAZEM ON STUNNED MYOCARDIUM EVALUATED WITH 99mTC-PYROPHOSPHATE IMAGING IN CANINE HEART
}

\author{
Ryuji Nohara, M.D., Hirofumi Kambara, M.D., Kazumi Okuda, M.D. \\ Shinji Ono, M.D., Nagara Tamaki, M.D.*, Jyunj Konishi, M.D* \\ and Chuichi Kawai, M.D.
}

\begin{abstract}
The effect of diltiazem on stunned myocardium was evaluated by measuring the myocardial uptake of 99m Tc-PYP (pyrophosphate) in open chest experiments with dogs. Myocardial stunning was induced by a $30 \mathrm{~min}$ ischemic occlusion of the anterior descending coronary artery. Regional wall motion was monitored by echocardiography of the epicardium for $2 \mathrm{~h}$ during reperfusion. After a 30 min occlusion of the coronary artery, it was reperfused and $99 \mathrm{~m}$ Tc-PYP was injected, followed by ${ }^{201} \mathrm{Tl} 2 \mathrm{~h}$ later. The ischemic area was defined by Evans blue dye, and the infarct area by TTC staining. No dogs showed infarcts or ${ }^{201} \mathrm{Tl}$ defects in this study group. Five dogs of the control-1 group $(\mathrm{C} 1$, ischemic area $=19.1 \pm 3.2 \%$ ) showed decreased regional wall motion during occlusion $(15.5 \pm 3.5 \%$ of control), and a slow recovery from depressed motion after $2 \mathrm{~h}$ of reperfusion $(20.3 \pm 9.3 \%)$ with uptake ratio (compared to the non-ischemic area uptake) of $99 \mathrm{~m}$ Tc-PYP $(4.96 \pm 2.28)$. In contrast, both groups with diltiazem infusion $(20 \mu \mathrm{g} / \mathrm{kg} / \mathrm{min})$, started either $30 \mathrm{~min}$ before ischemia (D1=5 dogs) or just after reperfusion (D2 $=5 \mathrm{dogs}$ ), showed significantly better recovery after $2 \mathrm{~h}$ of reperfusion (D1:115.4 $\pm 36.0 \%, \mathrm{D} 2: 109.2 \pm 44.2 \%$ ) than $\mathrm{C} 1$ $(\mathrm{p}<0.05)$, D1 and D2 groups also showed suppressed $99 \mathrm{mTc}-\mathrm{PYP}$ uptake ratio (D1:1.06 $\pm 0.33, \mathrm{D} 2: 2.34 \pm 2.05, \mathrm{p}<0.05$ vs $\mathrm{C} 1$ ) in spite of comparable ischemic area. Four dogs with small ischemic area $(\mathrm{C} 2: 5.3 \pm 5.0 \%)$ did not show increased $99 \mathrm{mTc}$-PYP uptake $(1.15 \pm 0.35)$, and regional wall motion after $2 \mathrm{~h}$ of reperfusion was $96.1 \pm 24.1 \%$ of the control value ( $p<0.05$ vs $C 1$ ). Thus, diltiazem was effective in enhancing the suppression of ${ }^{99 \mathrm{~m}}$ Tc-PYP uptake in the stunned myocardium, and similar results were obtained for small ischemic areas. The protective effect of diltilazem appears to be strongly related to the mechanism of $99 \mathrm{~m}$ Tc-PYP uptake.

(Jpn Circ J 1992; 56: 262-271)
\end{abstract}

$\mathbf{E}$ ARLY reperfusion of the occluded coronary artery may prevent myocardial injury or necrosis. However, the return of contractility in the myocardium salvaged

Key words:

Stunned myocardium

Diltiazem

99mTc-pyrophosphate by reperfusion may be delayed, and this phenomenon is termed "myocardial stunning"! The mechanisms of myocardial stunning are still controversial, $2-7$ and the clinical significance of myocardial stunning is also unclear. Several reports using $\mathrm{Ca}$ antagonists show favorable results in terms of

(Received April 20, 1991; accepted July 15, 1991)

3rd Division of Internal Medicine, and *Division of Nuclear Medicine, Kyoto University Hospital

This study was supported in part by the Grant-in-Aid for General Scientific Research from the Ministry of Education, Science and Culture, Japan

Mailing address: Ryuji Nohara, M.D., Third Division of Internal Medicine, Kyoto University Hospital, 54 Kawara-cho, Shogoin, Sakyo-ku, Kyoto city, Kyoto 606, Japan 


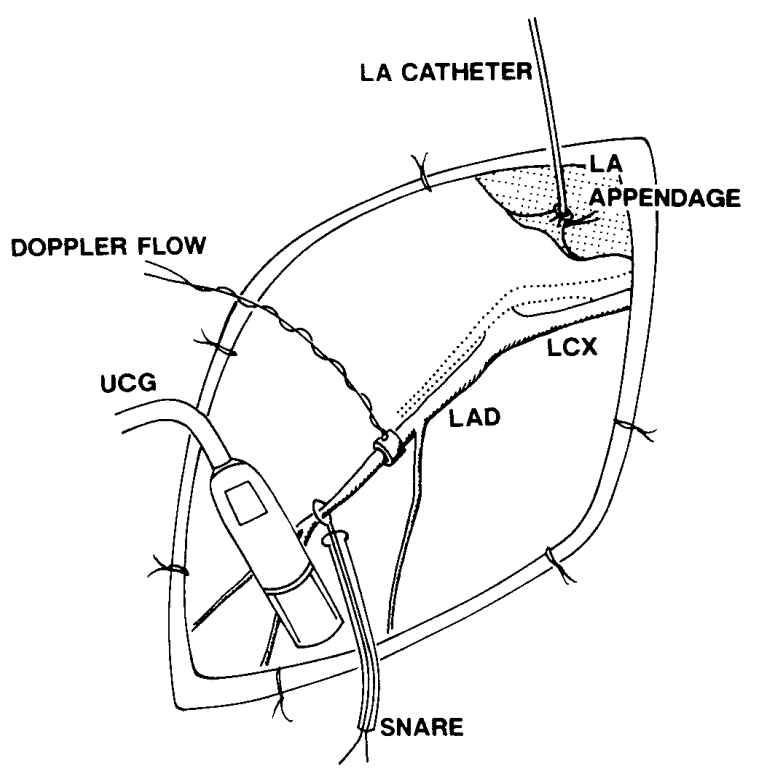

Fig.1. Schema showing an open chest dog experimental model. A doppler flow probe and snare were implanted in order around the left anterior descending artery (LAD) between the 1st and 2nd diagonal branche. Echocardiographic recording was performed from outside the epicardium, as shown in this figure.

preventing severe myocardial injury after ischemia8 Ca overloading is thought to be strongly related to reperfusion injury? This study deals with the stunned myocardium and evaluated the effect of $\mathrm{Ca}$ antagonists administered prior to and after ischemia. 99mTc-PYP (pyrophosphate) is commonly used for imaging and calculating the myocardial infarct size. We and others ${ }^{10}$ have found that severe ischemia without infarct is a strong stimulator of myocardial uptake of 99mTc-PYP. In this study, we report the favorable effects of the $\mathrm{Ca}$ antagonist, diltiazem on the stunned myocardium, with the inhibition of $99 \mathrm{mTc}$-PYP uptake. The mechanisms involved in myocardial stunning are also discussed.

\section{MATERIALS AND METHOD}

Adult mongrel dogs of either sex weighing 14 to $29 \mathrm{~kg}$ were subjected to an overnight fast, and were then anesthetized with 10.0 $\mathrm{mg} / \mathrm{kg}$ of ketaral i.m. and $25.0 \mathrm{mg} / \mathrm{kg}$ of sodium pentobarbital i.v. Animals were intubated and respirated with room air, and blood gases were kept within the physiological range. After intravascular instru- mentation, a left thoracotomy was performed at the fifth intercostal space and the heart was suspended in a pericardial cradle. A segment of the left anterior descending coronary between the first and second diagonal branch was gently dissected free. A doppler flow velocity probe and a snare were placed around the artery from proximal to distal in order, and catheter was inserted into the left appendage to inject isotope tracers (Fig. 1).

\section{Experimental protocol without drugs}

After stabilization of doppler flow, the following protocol was undertaken. A control echocardiograph was recorded with a 3.75 $\mathrm{MHz}$ transducer from the epicardium, and then the left anterior descending coronary artery (LAD) was ligated for 30 min with the snare. The ligated coronary artery was then reperfused with caution to prevent reactive hyperemia, and usually it took more than 5 min to reach to the control flow level. After doppler flow was stabilized, $20 \mathrm{mCi}(740$ $\mathrm{MBq}$ ) of $99 \mathrm{~m} \mathrm{Tc}$-pyrophosphate (99m Tc-PYP) was injected through the intra-atrial catheter. An echocardiographic recording was obtained just before reperfusion, and again $20 \mathrm{~min}, 1 \mathrm{~h}$ and $2 \mathrm{~h}$ after reperfusion. After $2 \mathrm{~h}$ of reperfusion $99 \mathrm{~m}$ Tc-PYP imaging was performed with a Searle gamma camera and analyzed with a DEC computer (PDP11/60). After ${ }^{99 m}$ Tc-PYP imaging, ${ }^{201}$ Tl was injected through the atrial catheter $(1-2$ $\mathrm{mCi}$ or $37-74 \mathrm{MBq}$ ) with subsequent imaging. To visualize the ischemic area, Evans blue dye $(10 \%, 100 \mathrm{ml})$ was injected through the atrial catheter just after ligation of the LAD with the same snare as used previously. Finally, saturated $\mathrm{KCl}$ was injected to sacrifice the dog.

The dog heart was removed with snare ligated, and cut into 4 or 5 slices perpendicular to the long axis of the heart, and as close as possible to the short axis of the echocardiographic recordings.

Heart slices were placed serally on a gamma camera, from the apex to the base, for ex-vivo imaging of $99 \mathrm{mTc}-\mathrm{PYP}$ and ${ }^{201} \mathrm{Tl}$. Peak energy was set at $140 \mathrm{KeV}$ with a $20 \%$ window for $99 \mathrm{mTc}$, and at $80 \mathrm{KeV}$ with a $20 \%$ window for ${ }^{201} \mathrm{Tl}$. There were 400.000 counts for in-vivo imaging, and 200,000 counts for ex-vivo imaging. Areas left 
TABLE I SUMMARY OF HEMODYNAMIC AND DOPPLER FLOW DATA

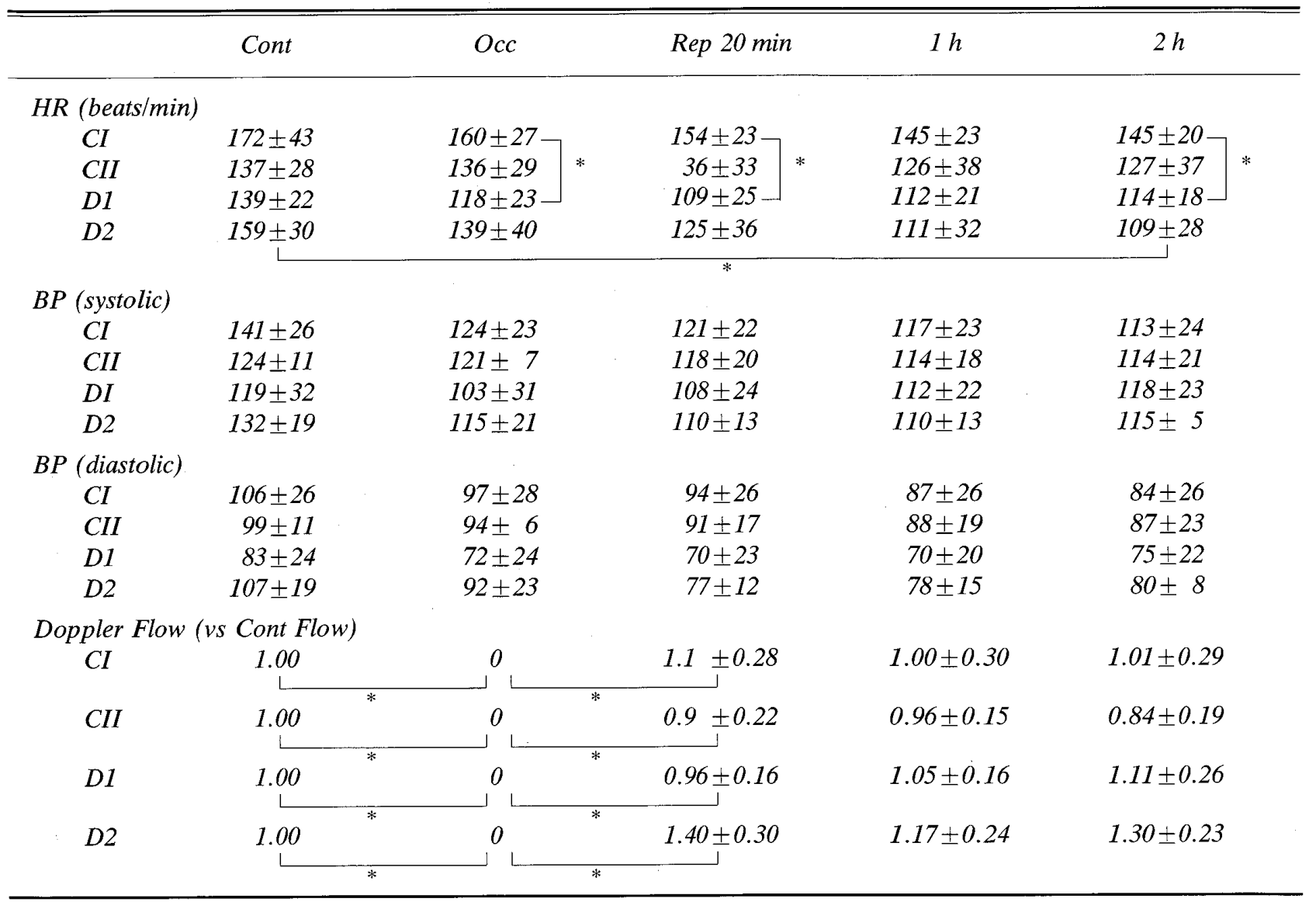

${ }^{*} p<0.05$, Cont $=$ control stage, Occ $=$ occlusion, Rep $20 \mathrm{~min}=20$ minutes after reperfusion, $1 \mathrm{~h}=1$ hour after reperfusion, $2 h=2$ hours after reperfusion.

unstained by Evans blue dye were considered as ischemic risk areas of the LAD region, and were traced onto translucent paper. Slices of the heart were then dipped into triphenyl-tetrazolium-chloride (TTC) in a solution of Sorensen's buffer for about 15 to $20 \mathrm{~min}$. Areas left unstained by TTC were thought to be infarcted, and were also traced. With reference to the risk area, ten small pieces $(0.5-1.0 \mathrm{~g}$ per piece) of the myocardium were excised from the endocardium and the epicardium in order to obtain tissue counts of $201 \mathrm{Tl}$ and $99 \mathrm{~m} \mathrm{Tc}$.

\section{Experimental protocol with drug administra- tion}

Diltiazem was administered either before occlusion of the coronary artery or immediately after reperfusion to investigate the protective effect of diltiazem on stunned myocardium. To study pre-medication, diltiazem $(20 \mu \mathrm{g} / \mathrm{kg} / \mathrm{min})$ was administered continuously through a peripheral line 30 min before occlusion, and was continued throughout the experiment. To study post medication, the same amount of diltiazem was infused just after reperfusion, and was continued until the end of the experiment.

Echocardiographic evaluation of wall motion Regional wall motion was evaluated by calculating the regional shortening of the endocardium by epicardial echocardiography. Short axial images at the level of papillary muscles were selected for calculation, and special attention was paid to keeping the position of the transducer constant during the experiments. Motion was measured along 100 chords constructed perpendicular to a centerline drawn midway between the end-diastolic and end-systolic contours with a computer. Deterioration of shortening was expressed as a \% of the control value, and the shortening of each point was expressed 


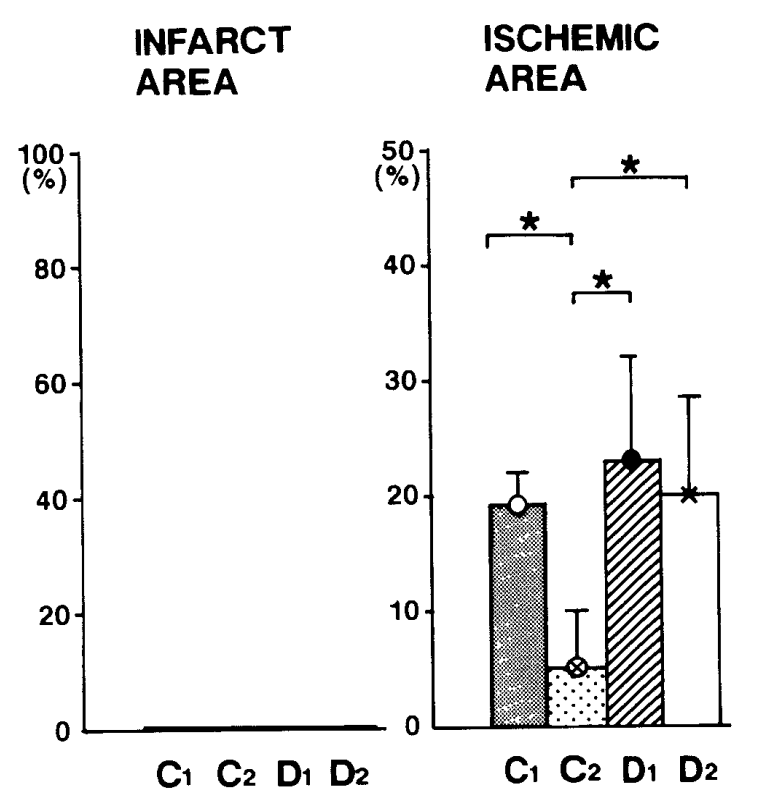

Fig.2. Infarct and ischemic areas in the study groups. No significant difference was present in either infarct or ischemic areas among groups C1, D1 and D2. However, the ischemic area of group C2 was significantly smaller than the other 3 groups $(\mathrm{p}<0.05)$.

in a circumferential profile. Chordal shortenings less than $50 \%$ of the control value were averaged as the shortening of the ischemic area.

\section{Evaluation of ischemic and infarct areas}

To evaluate the ischemic areas delineated by Evans blue dye, a non-stained area of each slice of the heart was traced and measured by a planimeter. Ischemic areas were calculated as a \% of the whole cut surface on both sides of each slice, and were averaged and multiplied by the weight of the slice. The total ischemic myocardial area was summed up and expressed as a \% of the left ventricle (LV) area. The same method was applied to calculate the infarct volume, as a $\%$ of the LV area.

\section{Tissue counting of ${ }^{99 m} T c-P Y P$ and ${ }^{201} \mathrm{Tl}$}

Tissue counting was performed by a well counter, with an acquisition time of one minute for each sample. Peak energies and windows were the same as for in-vivo imaging. The data were corrected for background and crossover counts. Tissue counts in the ischemic areas at the endocardium and epicardium were averaged, and expressed relative to the averaged non-ischemic area uptake (uptake ratio).

\section{Statistical analysis}

All data are expressed as mean values \pm SD. Analysis of variance with repeated values was used to compare responses between the groups. Students unpaired $t$ test was used for specific comparisons. A $\mathrm{p}$ value of less than 0.05 was considered significant.

\section{RESULTS}

Twenty-three dogs were studied, of which 3 died during the experiment, either coronary occlusion or just after reperfusion. One dog showed an anomalously big infarct by TTC staining in this $30 \mathrm{~min}$ occlusion-reperfusion model, and was excluded from the results. Thus, 19 non-infarcted dogs were evaluated for this study. Ten dogs out of 19 were treated with diltiazem. The 10 dogs with drug administration were divided into 2 groups: the premedicated group (5 dogs: Group D1) and the post-medication group (5 dogs: Group D2). The other 9 control dogs were also divided into 2 groups: one in which the ischemic area was more than $10 \%$ of the left ventricle (5 dogs: Group $\mathrm{C} 1$ ), and the other in which the ischemic area was less than $10 \%$ of the left ventricle. This group also exhibited fewer wall motion disturbances during occlusion (4 dogs: Group C2; explained in detail later).

\section{Heart rate, blood pressure and doppler flow (Table I) \\ Heart rates did not differ significantly dur-} ing the experiment among the groups, except in group D2 in which there was a significant difference between the control rate and the rate $2 \mathrm{~h}$ after reperfusion. Group $\mathrm{C} 1$ and group D1 showed a statistical difference at three sampling times, as shown in Table I, however, group $\mathrm{C} 1$ and group D2 did not. There was no difference in diastolic and systolic blood pressure between the groups. Doppler flow was expressed as a ratio of the control flow. During coronary occlusion, doppler flow was reduced to zero but returned to its control value after reperfusion in all groups. No statistical difference in doppler flow was seen among the 4 groups 


\section{REGIONAL WALL SHORTENING AND ${ }^{99 m}$ TC-PYP UPTAKE}
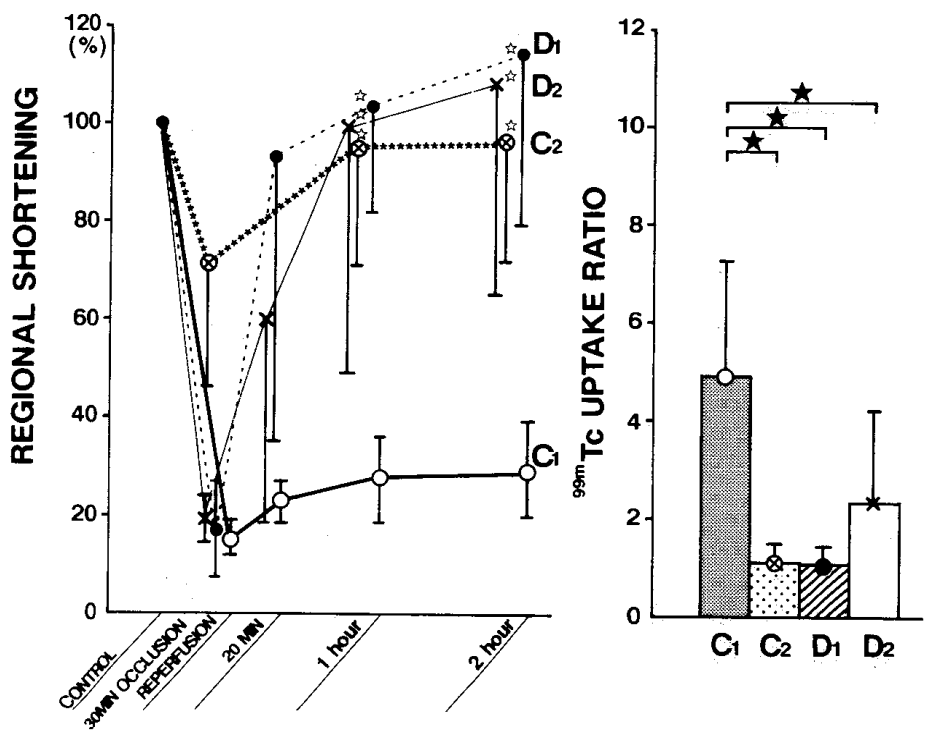

- DILTIAZEM PREMEDICATION $(\mathrm{N}=5)$

CONTROL1 $(\mathbf{N}=5)$

Q CONTROL2 $(\mathrm{N}=4)$

$\mathrm{p}<0.05$

$p<0.05$ vs $C_{1}$

Fig.3. Variability of regional wall motion among the 4 groups during the 5 stages of the study protocol. After $2 \mathrm{~h}$ of reperfusion, groups D1, D2 and $\mathrm{C} 2$ showed significantly better recovery than group C1. The right panel shows the $99 \mathrm{mTc}-\mathrm{PYP}$ uptake ratio for the 4 groups. Only group $C 1$ showed a high $99 \mathrm{mTc}$-PYP uptake ratio. Group D2 also tended to show a higher uptake than group C2 or D1.

at any experimental period.

\section{Ischemic and infarct area}

Figure 2 shows the ischemic and infarct areas, calculated with Evans blue and TTC staining, respectively. No infarct areas were observed in any groups. Ischemic areas were $19.1 \pm 3.2 \%$ of the LV in group C1, $23.8 \pm 9.1 \%$ in group D1 and $20.0 \pm 7.1 \%$ in group D2. Group C2 showed significantly less ischemic area $(5.3 \pm 5.0 \%)$ when compared to the other 3 groups $(\mathrm{p}<0.05)$.

\section{Regional wall motion with time}

Compared to the control value, regional shortening was attenuated at the end of the 30 min occlusion in group C1 $(15.5 \pm 3.5 \%)$, group D1 $(17.2 \pm 9.8 \%)$ and group D2 $(19.5 \pm 4.2 \%)$, without any significant differences among the 3 groups. After $20 \mathrm{~min}$ of reperfusion, groups D1 and D2 showed a remarkable improvement in regional wall motion (D1: $94.6 \pm 61.1 \%$, and D2: $60.1 \pm 42.5 \%)$ and tended to be far better than group $\mathrm{C} 1$ (C1: $15.5 \pm 3.5 \%)$. Group C1 showed minimal improvement after reperfusion. After 1 and $2 \mathrm{~h}$ of reperfusion, groups
D1 and D2 showed almost complete recovery and were significantly different $(\mathrm{p}<0.05)$ from group $\mathrm{C} 1$ ( $2 \mathrm{~h}$ value: $115.4 \pm 36.0 \%$ for $\mathrm{D} 1,109.2 \pm 442 \%$ for $\mathrm{D} 2$, and $20.3 \% \pm 9.3 \%$ for $\mathrm{C} 1$ ). Group C2 showed only slight deterioration in regional shortening during the occlusion, and recovered well after $1 \mathrm{~h}$ of reperfusion as shown in Fig. 3.

\section{${ }^{99 m}$ Tc-PYP uptake in the myocardium (Fig. 3)}

99m Tc-PYP uptake was evaluated by invivo and ex-vivo imaging. For in-vivo imaging, it was usually difficult to differentiate the ischemic areas from normal regions or adjacent organs such as bones or the lung if the relative uptake of $99 \mathrm{mTc}$ was less than 2.0. A relative uptake of more than 5.0 was easy to detect. In contrast, ex-vivo imaging showed abnormal $99 \mathrm{~m}$ Tc-PYP uptake as low as 1.2. Tissue counting showed a good, linear relationship with these findings. The uptake rate of $99 \mathrm{~m}$ Tc-PYP was $5.0 \pm 2.3$ in group $\mathrm{C} 1$, which was significantly different from group $\mathrm{C} 2 \quad(1.2 \pm 0.3 ; \mathrm{p}<0.05)$ and groups D1 and D2 $(1.1 \pm 0.3$ and $2.3 \pm 2.0$, respectively; $\mathrm{p}<0.05$ ). 


\section{REGIONAL SHORTENING}

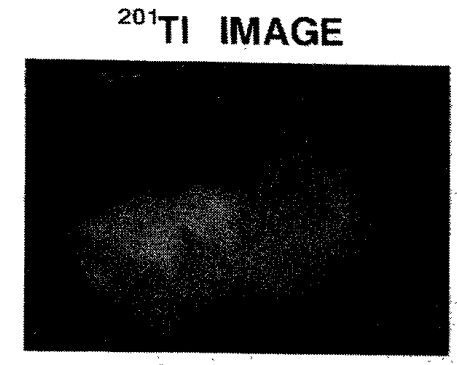

EX VIVO

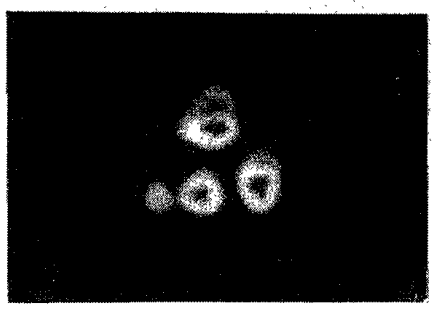

${ }^{99 m}$ TC IMAGE

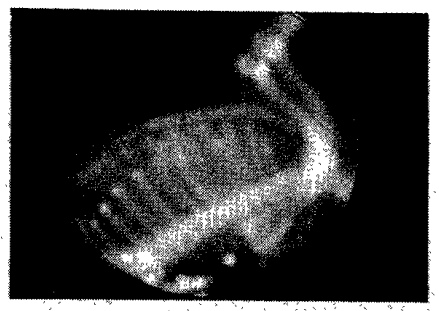

EX VIVO

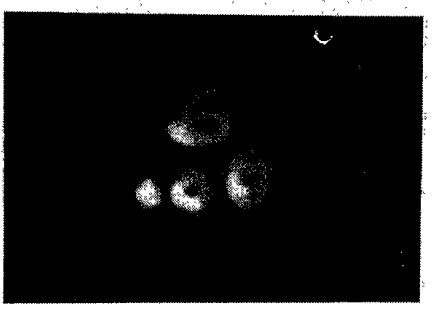

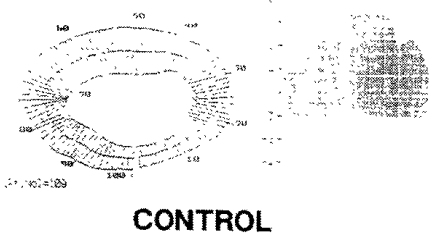

CONTROL

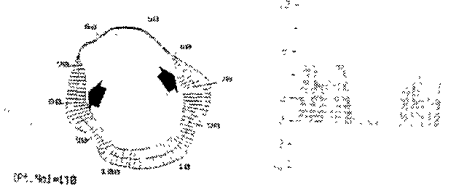

OCCLUSION

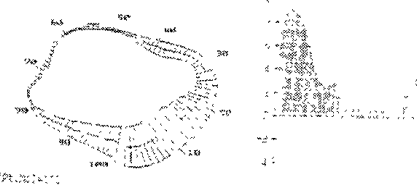

2H REPERFUSION

Fig.4. Ex-vivo and in-vivo images of ${ }^{201} \mathrm{Tl}$ and ${ }^{99 \mathrm{~m} T c-P Y P}$ uptake in a case without drug administration are shown. ${ }^{99 m}$ Tc-PYP imaging showed significantly higher uptake in the ischemic area (anterior wall) in spite of an absence of ${ }^{201} \mathrm{Tl}$ defect. Regional shortening calculated by echocardio graphy demonstrated a severe deterioration during occlusion, and poor recovery after $2 \mathrm{~h}$ of reperfusion. Wall motion was expressed by chordal display and circumferential profile methods.

\section{${ }^{201}$ Tl uptake in the myocardium}

${ }^{201} \mathrm{Tl}$ imaging showed diffuse uptake without any defects in both ex-vivo and in-vivo imaging. Tissue counting also showed no significant differences among the 4 groups. Relative uptake ratio was $2.5 \pm 1.9,0.9 \pm 0.1$, $0.9 \pm 0.2$, and $0.8 \pm 0.2$ for groups $\mathrm{C} 1, \mathrm{C} 2$, D1 and D2, respectively.

\section{Representative cases (Fig. 4 and 5)}

Case 1: This shows a representative case from group $\mathrm{C} 1$. Regional shortening of the echocardiographic chords showed deterioration in the anterior wall during occlusion and only slight recovery after $2 \mathrm{~h}$ of reperfusion. ${ }^{201} \mathrm{Tl}$ imaging in both ex-vivo and in-vivo experiments showed diffuse uptake without

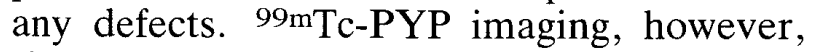
showed significant uptake in the ischemic areas.

Case 2: This shows a representative case from group D1. In spite of a severe reduction in regional shortening during occlusion, wall motion recovered after $2 \mathrm{~h}$ of reperfusion with diltiazem administration. Ex-vivo and in-vivo ${ }^{201} \mathrm{Tl}$ imaging showed no infarct and ${ }^{99 \mathrm{~m} T c-P Y P}$ uptake was suppressed.

\section{DISCUSSION}

Our data indicate that $99 \mathrm{~m}$ Tc-PYP uptake is closely related to recovery in the stunned myocardium. Recovery from the stunned myocardial state was almost complete with concomitant suppression of $99 \mathrm{~m}$ Tc-PYP uptake by diltiazem infusion, either premedicated or postmedicated, although the postmedicated group tended to show less suppression of 99mTc-PYP uptake and poorer recovery after $20 \mathrm{~min}$ of reperfusion. This small difference of recovery process was not significant, because of the large standard deviation (SD) from mean, however, the same final quantitative value of recovery suggests that the recovery for either method of medication with diltiazem will be similar 


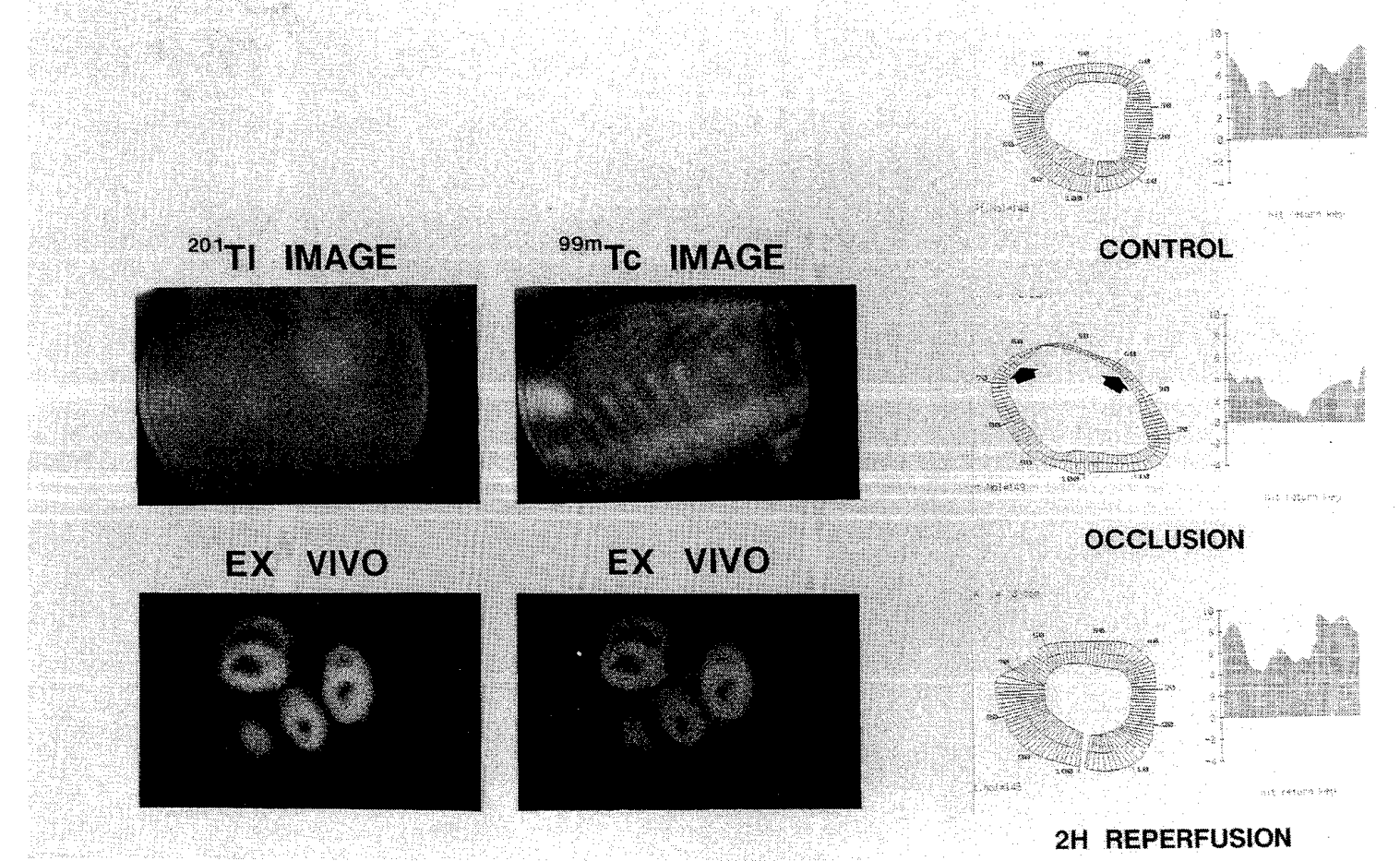

Fig.5. A case where diltiazem infusion started $30 \mathrm{~min}$ before occlusion. No ${ }^{99 m T c-P Y P}$ uptake was noted, and good recovery of regional shortening was demonstrated.

finally even if acceleration of recovery in the early phase of reperfusion may show some difference between them. The deterioration of the regional wall motion during occlusion was slight, and improvement was complete in cases with little ischemia, and ${ }^{99 \mathrm{mTc}-P Y P}$ uptake and recovery from ischemia resembled the responses found for the pre-and post-medicated groups.

\section{${ }^{99 m}$ Tc-PYP uptake and ischemia without infarct}

The localization of free calcium within the damaged myocardial cell has been postulated as a reason for the uptake of bone imaging agents (99m Tc-PYP) in acute myocardial infarction. Available data has shown that calcium deposits in irreversibly injured muscle cells are localized primarily in the mitochondira in the form of apatite like spicules or subcrystalline, fine granular, amorphous calcium. ${ }^{99 \mathrm{~m} T c-l a b e l e d}$ pyrophosphate agents exhibit a similar distribution pattern to calcium!11,12 Buja and co-workers reported numerous contraction bands and calcium deposits in the outer periphery of the infarct where the pyrophosphate uptake was high!2
A close correlation was also found between the subcellular uptake of $\left[{ }^{99 \mathrm{~m} T c}\right]$ pyrophosphate and that of calcium ${ }^{13}$ suggesting elevated intracellular calcium level plays a key role in myocardial ${ }^{99 \mathrm{~m} T c-P Y P}$ scintigraphy in a chemical milieu induced by ischemia. Infarct size has been measured accurately in dogs, especially in acute anterior and lateral infarcts ${ }^{14}$ with ${ }^{99 m}$ Tc-PYP. This has been broadly applied to humans. However, positive myocardial scintigrams with $99 \mathrm{~m}$ Tc-PYP in patients with "unstable angina" suggest that there may be limited myocardial necrosis that is ordinarily undetectable by ECG and enzyme assays ${ }^{15}$ Several other reports regarding unstable angina also suggest that ischemia without infarct may induce increased intracellular concentrations of ${ }^{99 \mathrm{~m} T c-P Y P} 16,17$

By a use of an isolated-heart preparation, $99 \mathrm{~m}$ Tc glucoheptonate uptake was evaluated as an infarct marker by Horner ${ }^{18}$ The uptake ratio between ischemic tissue samples and the normal myocardium was 4.5 , after 25 min of occlusion followed by reperfusion. However, no infarcts were found by nitro blue tetrazolium (NBT) staining!9 It takes 
more than 40 min to consistently produce infarcts in the isolated canine heart ${ }^{20}$ In an experimental canine model with a 40 min acute coronary occlusion followed by a $6 \mathrm{~h}$ reperfusion, Biance showed that areas that were ischemic during occlusion had a normal flow after reperfusion and were viable as demonstrated by TTC staining, with a twofold increase in $99 \mathrm{mTc}$-PYP uptake when compared to non-ischemic regions ${ }^{10}$

Thus, clinical as well as experimental studies have shown that ischemia without infarct seems to increase the intra-cellular uptake of ${ }^{99 m}$ Tc-PYP and appears to be closely associated with calcium metabolism.

\section{Ischemia and the stunned myocardium}

Experimental studies have demonstrated that early reperfusion limits or even prevents necrosis. However, these beneficial effects do not lead to immediate functional recovery, but rather lead to a delayed recovery of contractility that extends for hours, days or even weeks. This phenomenon is termed "myocardium stunning". Numerous mechanisms have been proposed thus far, including heterogenous impairment of myocardial perfusion', abnormalities of myocellular excitation, loss of myofibrillar creatine kinase activity, ${ }^{4}$ cytotoxic effects of free radicals, functional sympathetic denervation $^{6}$ and altered calcium homeostasis resulting in defective excitation-contraction coupling? Our canine model of a 30 min occlusion-reperfusion without infarct is in agreement with the condition of "stunned myocardium", 1 since none of the dogs showed any myocardial necroses except one (which was excluded due to the presence of an abnormally large infarct defined by TTC).

\section{Effect of the calcium antagonist on post-ische- mic "stunned myocardium"}

In a canine experimental model of a 15 min occlusion-reperfusion, administration of verapamil $30 \mathrm{~min}$ before occlusion or even after reperfusion significantly increased contractility of the post-ischemic myocardium but did not influence storage of high energy phosphate.2 Calcium antagonists, including verapamil, have the effect of systemic vasodilation with reduced afterload as well as of coronary vasodilation, both of which will in- crease regional function. However, blood pressure was not significantly different from the control valued in our dog model, and neither was the doppler flow. HR reduction may not be the primary cause of protection with diltiazem, because both D1 and D2 showed the same inhibition of $99 \mathrm{~m} \mathrm{Tc}-$ PYP and improvement of wall motion although HR differed only in group D1 from group $\mathrm{C} 1$ in some sampling times. We did not measure the regional flow with microspheres during or after reperfusion, however, the ischemic areas were clearly defined by Evans blue and the regional function was also assessed. In addition, ${ }^{201} \mathrm{Tl}$ scintigraphic and tissue counting studies showed normal radioactivity in all of the dogs including group $\mathrm{C} 1$, in spite of depressed regional function $2 \mathrm{~h}$ after reperfusion. Therefore, improved myocardial perfusion may not be the major mechanism by which diltiazem improves regional function after reperfusion. The application of ${ }^{201} \mathrm{Tl}$ scintigraphy for the evaluation of perfusion and viability in the stunned myocardium was tested by Moore et al,23 and it was reported that ${ }^{201} \mathrm{Tl}$ kinetics and washout were normal in canine experiments. The normal kinetics of ${ }^{201} \mathrm{Tl}$ after brief ischemia strongly support our hypothesis that recovery from myocardial stunning due to diltiazem is not due to improved perfusion in the ischemic area.

Apart from these advantageous effects of calcium antagonist, our results strongly suggest a specific action of calcium channel blocking agents on myocytes to improve regional function. Recent studies of both global $^{24}$ and regional ischemia 25 indicate that calcium transport and flux within myocytes is impaired after a brief ischemic episode. This alteration in calcium homeostasis may affect mitochondrial function and high energy phosphate production, and the calcium flux may trigger the production of cytotoxic free radicals? Thus, modulation of calcium influx and homeostasis may be a major mechanism by which calcium antagonists affect stunned myocardium.

\section{Effect of diltiazem on $99 m$ Tc-PYP uptake after ischemia}

$\mathrm{Ca}$ antagonists probably block all Ca-channels, or actually enter the cell, where they 
substitute for $\mathrm{Ca}^{2+}$ at intracellular receptors. The two significant properties of $\mathrm{Ca}$ antagonists are; 1) the ability to block the slow calcium channel in the cardiac muscle, and 2) the ability to inhibit transmembrane fluxes of calcium in the smooth muscle, especially in the coronary and peripheral circulations ${ }^{26}$ Pretreatment with diltiazem is known to suppress $\mathrm{Ca}$ overload, a well kown cause of myocardial injury during reperfusion? This results in a reduction of infarct size, and maybe related to stunning. ${ }^{27}$

Reperfusion with low $\mathrm{Ca}$ buffers attenuated the depression of myocardial function and stunning? ${ }^{27} \mathrm{Ca}$ entry following overloading in the cell was shown to be strongly related to stunning, Thus, diltiazem may work as a stunning inhibitor by blocking $\mathrm{Ca}^{2}+$ entry. ${ }^{99 \mathrm{~m} T c-P Y P}$ distributes in the same fashion as $\mathrm{Ca}^{2}+$ in the cell, and the decreased inward movement of $99 \mathrm{mTc}-\mathrm{PYP}$ may be indicative of depressed cellular $\mathrm{Ca}$ overloading and stunning. Thus, diltiazem may work as an inhibitor of $\mathrm{Ca}$ influx or of $\mathrm{Ca}$ overloading, and may be helpful in the recovery form stunning.

\section{Clinical implication}

Either premedication with diltiazem or administration after the ischemic episode was effective in obtaining early recovery from stunning with suppression of $99 \mathrm{~m}$ TcPYP uptake. This implies that diltiazem administration, either before or early after revascularization procedures such as thrombolysis or percutaneous transluminal coronary angioplasty, might be affective in speeding for early recovery from depressed wall motion. In addition, $99 \mathrm{~m} T \mathrm{Tc}-\mathrm{PYP}$ uptake does not necessarily indicate a myocardial infarction but may rather indicate a reversible ischemia. The uptake can be significantly modified by medication, such as diltiazem. Although the relationship between myocardial stunning and $99 \mathrm{mTc}-\mathrm{PYP}$ uptake remains to be elucidated, the radionuclide assay method may become a valuable in-vivo procedure to understand the mechanism and inhibition of myocardial stunning.

\section{Acknowledgment}

We thank Todd Crick and Bruce Symons for their careful correction and preparation of the typescript.

\section{REFERENCES}

1. BRAUNWALD E, KLONER RA: The stunned myocardium: Prolonged, postischemic ventricular dysfunction. Circulation 1982; 66: $1148-1149$

2. STAHL LD, AVERSANO TR, BECKER LC: Selective enhancement of function of stunned myocardium by increased flow. Circulation 1986; 74: $843-851$

3. LOBINE JH, MOORE EN, WEISMAN HF, KADISH AH, BECKER LC, SPAR JF: Depression of action potential characteristics and a decreased space constant are present in postischemic, reperfused myocardium. J Clin Invest 1987; 79: 107 $-116$

4. GREENFIELD RA, SWAIN JL: Disruption of myofibrillar energy use: Dual mechanisms that may contribute to postischemic dysfunction in stunned myocardium. Circ Res 1987; 60: 283-289

5. McLORD JM: Oxygen-derived free radicals in postischemic tissue injury. $N$ Engl J Med 1985; 312: $159-163$

6. CIUFFO AA, OUYANG P, BECKER LC, LEVIN L, WEISFELDT ML: Reduction of sympathetic inotropic response after ischemia in dogs; Contributor to stunned myocardium. J Clin Invest 1985; 75: $1504-1505$

7. ITO BR, TATE H, KOBAYASHI M, SCHAPER W: Reversibly injured, postischemic canine myocardium retains normal contractile reserve. Circ Res 1987; 61: 834-846

8. KLEIN HH, SCHUBOTHE M, NEVENDAHL K, KREUZER H: The effects of two different diltiazem treatment on infarct size in ischemic, reperfused porcine hearts. Circulation 1984; 69: $1000-1005$

9. SHINE KL, DOUGLAS AM: Low calcium reperfusion of ischemic myocardium. J Moll Cell Cardiol 1983; 15: 251-260

10. BIANCO JA, KEMPER AJ, TAYLOR A, LAZEWATSKY J, TOW DE, KHURI SF: Technetium-99m $\left(\mathrm{Sn}^{2+}\right)$ pyrophosphate in ischemic and infarcted dog myocardium in early staged of acute coronary occlusion: Histochemical and tissuecounting comparisons. $J \mathrm{Nucl}$ Med 1983; 24: $485-491$

11. BUJA LM, PARKEY RW, DEES JH, et al: Morphologic correlates of technetium-99m stannous pyrophosphate imaging of acute myocardial infarcts in dogs. Circulation 1975; 52: 596-607

12. BUJA LM, TOFE AJ, KULKARNI PV, et al: Site and mechanisms of localization of technetium$99 \mathrm{~m}$ pyrophosphate radiopharmaceuticals in acute myocardial infarcts and other tissues. J Clin Invest 1977; 60: $724-740$

13. BUJA LM, TOFE AJ, MUKHERJEE A, et al: Role of elevated tissue calcium in myocardial infarct scintigraphy with technetium phosphorus radiopharmaceuticals, abstracted. Circulation 1976; 54(Suppl 2): 219

14. STOKELY EM, BUJA LM, LEWIS SE, et al: Measurement of acute myocardial infarcts in dogs with $99 \mathrm{~m}$ Tc-stannous pyrophosphate scintigrams. $J$ Nucl Med 1976; 17: 1-5

15. WILLERSON JT, PARKEY RW, BONTE EJ, 
MEYER SL, ATKINS JM, STOKELY EM: Techmetium stannous pyrophosphate myocardial scintigrams in patients with chest pain of varying etiology. Circulation 1975; 51: 1046-1052

16. ABDULLA AM, CANEDO MI, CORTEZ BC, McGINNIS KD, WILHELM SK: Detection of unstable angina by $99 \mathrm{~m}$-technetium pyrophosphate myocardial scinigraphy. Chest 1976; 69: 168-173

17. LESSEM J, JAHANSSON BW, NOSSLIN B, THORELL J: Myocardial scintigraphy with $99 \mathrm{mTc}$ pyrophosphate in patients with unstable angina pectoris. Acta Med Scand 1978; 203: 491-495

18. JACOBSTEIN JM, ALONSO DR, ROBERTS AJ, CIPRIANO PR, COMBES JR, POST MR: Early diagnosis of myocardial infarction in the dog with 99mTc-luconeptonate. J Nucl Med 1977; 18: 413-418

19. HONER WH, ALONSO DR, JACOBSTEIN JG: Ischemia and infarction in the isolated rabbit heart: A model for the evaluation of myocardial imaging agents. J Nucl Med 1980; 21: 523-528

20. JENNINGS RB, SOMMERS HM, SMYTH GA, FALCK HA, LINN H: Myocardial necrosis induced by temporary occlusion of a coronary artery in the dogs. Arch Pathol 1960; 70: 68-78

21. JENNINGS RB, REIMER KA: Factors involved in salvaging ischemic myocardium: Effect of reperfusion of arterial blood. Circulation 1983; 68(Supple 1): I-25-6
22. PRZYKLENK K, KLONER RA: Effect of verapamil on postischemic "stunned" myocardium: Importance of the timing of treatment. $J \mathrm{Am}$ Coll Cardiol 1988; 11: 614-623

23. MOORE CA, CANNON J, WATSON DD, KAUL SK, BELLER GA: Thallium-201 kinetics in stunned myocardium characterized by severe postischemic systolic dysfunction. Circulation 1990; 81: $1622-1632$

24. KRAUSE SM, HESS ML: Characterization of cardiac sarcoplasmic reticulum dysfunction during short-term, normothermic, global ischemia. Circ Res 1984; 55(2): 176-184

25. KRAUSE SM, JACOBS WE, BECKER LC: Alteration in sarcoplasmic reticulaum $\mathrm{Ca}^{2}+$ transport in the post-ischemic "stunned" myocardium. Circulation 1986; 74(Suppl 11): II-67

26. SINGH BN, HECHT HS, NADEMANEE K, CHEW CYC: Electrophysiologic and hemodynamic effects of slow-channel blocking drugs. Prog Cardiovasc Dis 1982; 23: 103-132

27. KUSUKAWA H, PORTERFIELD JK, WEISMAN HF, WESFELDT NL, MARBAN E: Pathophysiology and pathogenesis of stunned myocardium; Depressed $\mathrm{Ca}^{2+}$ activation of contraction as a consequence of reperfusion-induced cellular calcium overload in ferret hearts. $J$ Clin Invest 1987; 79: 950-961 